\title{
MINIMAL INVASIVE SURGICAL TECHNIQUES FOR \\ THE TREATMENT OF PATHOLOGIC LESIONS, SITUATED IN THE MIDLINE OF THE SPINAL CANAL
}

\author{
Zoltán Papp ${ }^{1}$, János Vajda ${ }^{1}$, Róbert Veres ${ }^{2}$, Péter Banczerowski ${ }^{1}$ \\ ${ }^{1}$ National Institute of Neurosurgery, Budapest, Hungary \\ ${ }^{2}$ Department of Neurosurgery ÁEK, Budapest, Hungary \\ zolpapp@oiti.hu
}

\begin{abstract}
Objective: Multilevel laminectomy for exposing the spinal canal to remove spinal cord lesions has been widely used in spine surgery. Destruction of the dorsal structures of the spinal column, detachment of the longitudinal musculature, resection of the vertebral arches, and injury of the joint capsules and ligaments are responsible most of short and late-time complications. Spinal deformities, instability, subluxation, invasion of haematoma and scar tissue into the spinal canal are the most often mentioned complications in the literature. The author main objective was to develop and summarize the novel minimally invasive techniques suitable for exploring and treating different pathologies, located in the midline of the spinal canal with preservation of the stability of the spine.
\end{abstract}

Methods: 38 patients were operated on with intramedullary laesions located from CIII to LI level of the spine with the newly developed multilevel spinous process splitting and distracting laminotomy technique. The dorsal, paraspinal musculature was not detached from the vertebras. With splitting and distracting the spinous processes and the vertebral arches, the vertebral joints, the joint capsules and the ligaments were not injured, these structures remained mostly intact. To achieve a moderate enlargement and decompression of the spinal canal, complementary intervertebral spacer insertion was performed in some cases. The patients were followed with regular MRI, CT scans and neurological examinations.

Results: Adequate surgery of the lesions located intramedullary was achieved in all patients using our new procedures. Moderate enlargement and permanent decompression of the spinal canal was achieved with the insertion of homologues tricortical iliac crest bone graft or heterologous PEEK spacer. The numbers of split laminae were 3 to 6 . The split spinous processes were closed directly to each other in 24 patients. In 9 cases a tricortical bone graft and in 5 cases a heterologous PEEK spacer was inserted between the facing bony parts.

The incidence of postoperative local pain was lower, within acceptable limits, and early mobilization was allowed. The average length of hospital stay was shorter too. The postoperative followup CT scans demonstrated bony healing, with the inserted graft or cage between the osteotomized faces. No compression or dislocation of the spacer was seen. Instability was not detected in any of the patients by flexion or extension lateral radiographs. 
Conclusion: The split laminotomy surgical procedure with or without complementary auto- or heterologous grafting method fulfills the requirements of other laminotomy techniques. This technique is suitable for removing intramedullary tumors, and the posterior stabilizing structures of the spine, as the vertebral laminae and the longitudinal musculature are completely preserved, nearly anatomical situation can be maintained. Leaving the longitudinal paraspinal musculature innervations intact, and with the preservation of the bone-muscle attachments and ligaments, the dynamic stability of the spine remains unchanged. Retaining the bony structures (vertebral arches) and the vertebral joints the static stability of the spinal column remain intact, the chance of developing the long-term spinal deformation is minimal. The grafts, inserted between the osteotomized faces, provided permanent decompression of the spinal canal, and bony healing - throughout the graft or spacer - of the split vertebral laminae. With the use of the PEEK spacer the short and long time complications of the autologous bone graft harvesting procedure can be avoided.

This newly developed surgical procedure and its modifications can be used at any age of the patients, any level of the spine, theoretically on unlimited number of spinous processes.

Keywords: laminotomy; splitting laminotomy; intramedullary tumor; intervertebral spacer

\section{Introduction}

Multilevel laminectomy for exposing the spinal canal to remove spinal cord lesions has been widely used in spine surgery ${ }^{4,7}$. Many of short and late-time complications of this surgical procedure have been reported. Spinal deformities, instability, subluxation, invasion of haematoma and scar tissue into the spinal canal are the most often mentioned complications in the literature ${ }^{6,8,20}$. Several surgical procedures have been reported to preserve the posterior structures. Various kinds of laminoplasty techniques have been described with osteoplastic posterior spinal arch reconstruction, in tumor removal and in degenerative cases as well ${ }^{7,11}$. The conventional posterior surgical approaches invariably separate the muscle attachments from the spinous processes and laminae. Damage to these muscles and bony connections can lead to persistent axial pain, cervical malalignment and spinal instability ${ }^{10}$. Postlaminectomy kyphotic deformation is one of the most known long-term complications of the classic dorsal surgical procedures. The operative treatment of this deformation is very difficult and less effective.
To preserve the dynamic and static stabilizing structures of the spinal column new minimally invasive ways to explore the spinal canal have been developed. To follow the principle of less invasiveness the split laminotomy technique for surgery of multilevel lesions located in the spinal canal was introduced ${ }^{1,9}$. This surgical procedure is suitable for exploring and removing different pathologies located in the spinal canal. It has been proven, that the split laminotomy approach is suitable to remove intramedullary tumors located in the midline.

If total resection of an intramedullary tumor was not possible due to the infiltration to the surrounding spinal cord or re-growing of the malignant tumor is expectable, an enlargement of the spinal canal is needed. To achieve the permanent enlargement of the spinal canal and decompression of the spinal cord, a bone graft or PEEK cage implantation was performed between the split laminas. The bone grafting procedure is a well developed and widely used method in spine surgery. The tricortical iliac bone graft is most commonly used at ventral cervical discectomy or cervical 
corpectomy procedures $2,3,5,13$. To avoid the short and long-term bone harvesting area complications, and to shorten the time of the surgical procedure, the use of different interbody spacers is widely accepted at spinal surgical procedures. With the cage implantation all of the donor site complications, and the possibility of late-time bone graft resorption or compression is avoidable $12,14,15,16$. The bony healing throughout the spacer is similar to the iliac crest bone graft procedure ${ }^{17,18}$. Solid fusion between the osteotomized parts can be shown about 12 months after the implantation.

\section{Clinical Materials and Methods}

The authors used the multilevel spinous process splitting and distracting laminotomy technique with or without complementary auto,or allograft insertion in 38 adult patients with intramedullary lesions, located in various level of the spinal cord. The split spinous processes were closed directly to each other in 24 patients (Group I). In 9 cases a tricortical iliac crest bone graft (Group II/A) and in 5 cases a heterlogous Poly-Ether-Ether-Ketone (PEEK) intervertebral spacer (Group II/B) was inserted between the facing bony parts (Table 1).

\begin{tabular}{|c|c|c|c|c|c|c|c|}
\hline $\begin{array}{c}\text { Patient } \\
\text { no. }\end{array}$ & $\begin{array}{c}\text { Age }(y r) / \\
\operatorname{sex}\end{array}$ & Histology & $\begin{array}{l}\text { Resection } \\
\text { (MRI) }\end{array}$ & $\begin{array}{l}\text { No. of split } \\
\text { laminae }\end{array}$ & Group & $\begin{array}{l}\text { Preoperative } \\
\text { functional } \\
\text { assessment }\end{array}$ & $\begin{array}{l}\text { Postoperative } \\
\text { functional } \\
\text { assessment }\end{array}$ \\
\hline 1 & $44 / \mathrm{M}$ & Astrocytoma Gr III & partial & 3 & II/A & II & II \\
\hline 2 & $59 / \mathrm{M}$ & Ependymoma & complete & 4 & $\mathrm{I}$ & II & I \\
\hline 3 & $47 / \mathrm{F}$ & Ependymoma & complete & 4 & I & I & I \\
\hline 4 & $55 / \mathrm{F}$ & Astrocytoma Gr II & subtotal & 5 & $\mathrm{I}$ & III & III \\
\hline 5 & $61 / \mathrm{F}$ & Astrocytoma Gr III & partial & 3 & II/A & $\mathrm{I}$ & II \\
\hline 6 & $44 / \mathrm{F}$ & Ependymoma & partial & 3 & $\mathrm{II} / \mathrm{A}$ & I & I \\
\hline 7 & $52 / \mathrm{M}$ & Astrocytoma Gr II & partial & 5 & II/A & II & II \\
\hline 8 & $61 / \mathrm{M}$ & Astrocytoma Gr I & complete & 6 & $\mathrm{I}$ & II & II \\
\hline 9 & $46 / \mathrm{M}$ & Ependymoma & partial & 3 & II/A & I & II \\
\hline 10 & $55 / \mathrm{F}$ & Cavernoma & complete & 3 & $\mathrm{I}$ & II & $\mathrm{I}$ \\
\hline 11 & $49 / \mathrm{F}$ & Ependymoma & complete & 4 & $\mathrm{I}$ & $\mathrm{I}$ & $\mathrm{I}$ \\
\hline 12 & $58 / \mathrm{M}$ & Astrocytoma Gr II & subtotal & 3 & $\mathrm{I}$ & II & III \\
\hline 13 & $38 / \mathrm{M}$ & Astrocytoma Gr I & complete & 4 & $\mathrm{I}$ & $\mathrm{I}$ & $\mathrm{I}$ \\
\hline 14 & $57 / \mathrm{M}$ & Ependymoma & partial & 5 & II/A & II & II \\
\hline 15 & $56 / \mathrm{F}$ & DAVF & closed & 3 & $\mathrm{II} / \mathrm{A}$ & II & $\mathrm{I}$ \\
\hline 16 & $48 / \mathrm{F}$ & Ependymoma & complete & 4 & I & $\mathrm{I}$ & $\mathrm{I}$ \\
\hline 17 & $43 / \mathrm{F}$ & Ependymoma & complete & 4 & I & $\mathrm{I}$ & I \\
\hline 18 & $60 / \mathrm{F}$ & Astrocytoma Gr III & partial & 3 & II/A & II & II \\
\hline 19 & $55 / \mathrm{M}$ & Cavernoma & complete & 5 & $\mathrm{I}$ & I & $\mathrm{I}$ \\
\hline 20 & $47 / \mathrm{F}$ & Cavernoma & complete & 3 & I & I & $\mathrm{I}$ \\
\hline 21 & $52 / \mathrm{M}$ & Ependymoma & complete & 3 & I & I & I \\
\hline 22 & $59 / \mathrm{M}$ & Astrocytoma Gr II & complete & 3 & I & II & II \\
\hline 23 & $52 / \mathrm{F}$ & Ependymoma & complete & 5 & I & I & I \\
\hline 24 & $43 / \mathrm{M}$ & Astrocytoma Gr III & partial & 6 & II/A & II & II \\
\hline 25 & $46 / \mathrm{F}$ & AVM & closed & 3 & II/B & I & I \\
\hline 26 & $57 / \mathrm{M}$ & Ependymoma & complete & 4 & $\mathrm{I}$ & $\mathrm{I}$ & $\mathrm{I}$ \\
\hline 27 & $54 / \mathrm{M}$ & Haemangioblastoma & complete & 3 & $\mathrm{I}$ & II & II \\
\hline 28 & $47 / \mathrm{F}$ & Astrocytoma Gr II & complete & 5 & I & II & III \\
\hline 29 & $59 / \mathrm{F}$ & Astrocytoma Gr III & partial & 3 & II/B & II & II \\
\hline 30 & $56 / \mathrm{M}$ & Ependymoma & complete & 3 & $\mathrm{I}$ & $\mathrm{I}$ & $\mathrm{I}$ \\
\hline 31 & $47 / \mathrm{F}$ & Ependymoma & complete & 4 & I & I & $\mathrm{I}$ \\
\hline 32 & $41 / \mathrm{M}$ & Astrocytoma Gr II & subtotal & 5 & $\mathrm{I}$ & II & III \\
\hline 33 & $50 / \mathrm{F}$ & Astrocytoma Gr II & partial & 4 & II/B & II & III \\
\hline 34 & $57 / \mathrm{M}$ & DAVF & closed & 3 & $\mathrm{II} / \mathrm{B}$ & I & I \\
\hline 35 & $52 / \mathrm{M}$ & Astrocytoma Gr III & partial & 5 & $\mathrm{II} / \mathrm{B}$ & II & III \\
\hline 36 & $45 / \mathrm{M}$ & Ependymoma & complete & 6 & I & I & I \\
\hline 37 & $61 / F$ & Ependymoma & complete & 6 & $\mathrm{I}$ & I & I \\
\hline 38 & $44 / \mathrm{M}$ & Ependymoma & complete & 4 & I & I & I \\
\hline
\end{tabular}

Table 1. Characteristics of the patients 
There were 18 women and 20 men with an average age of 51.6 years (range 38-63 years) at the time of surgery. (Table 1) Functional assessment (McCormic) was performed preoperatively and postoperatively every 6 months at the time of the MRI follow-up visits. To confirm the extension of resection and to check for recurrence or to follow the growing patterns of the tumor, all patients underwent postoperative MRI evaluations at 3 and 6 months postoperatively, thereafter every 6 months or as needed by the patient's condition. To check the bony changes, all patients had postoperative CT imaging as well immediately after the operation and repeatedly thereafter (at 2, 6, and 12 months).

The patients were positioned sitting or prone for cervical and prone for thoracic and thoracolumbar surgeries. A special midline posterior approach was used. The skin, fascia, and nuchal in the cervical region and the supraspinous ligament in the midline were incised.
The interspinous ligaments and muscles were dissected longitudinally between the spinous processes without injuring the attachments of the interspinous muscles. All muscle attachments on the spinous processes and laminas, as well as the laminas themselves, were completely preserved. The innervations of the longitudional paravertebral musculature left completely intact. The vertebral joints, and the capsules were also not injured. The ligamentum flavum was removed at the middle part to expose the midline epidural space above and below the intended levels. In some cases involving the upper and midthoracic region of the spine, it was necessary to remove the cephalad small bony part of the angle of the vertebral arcuses in the midline as a result of the oblique location of the spinous processes. The spinous processes were split in the midline with an oscillating saw or craniotome. The spinous processes and the laminae were separated and distracted with Cloward-type retractors (Figure 1). It is important to accurately fit

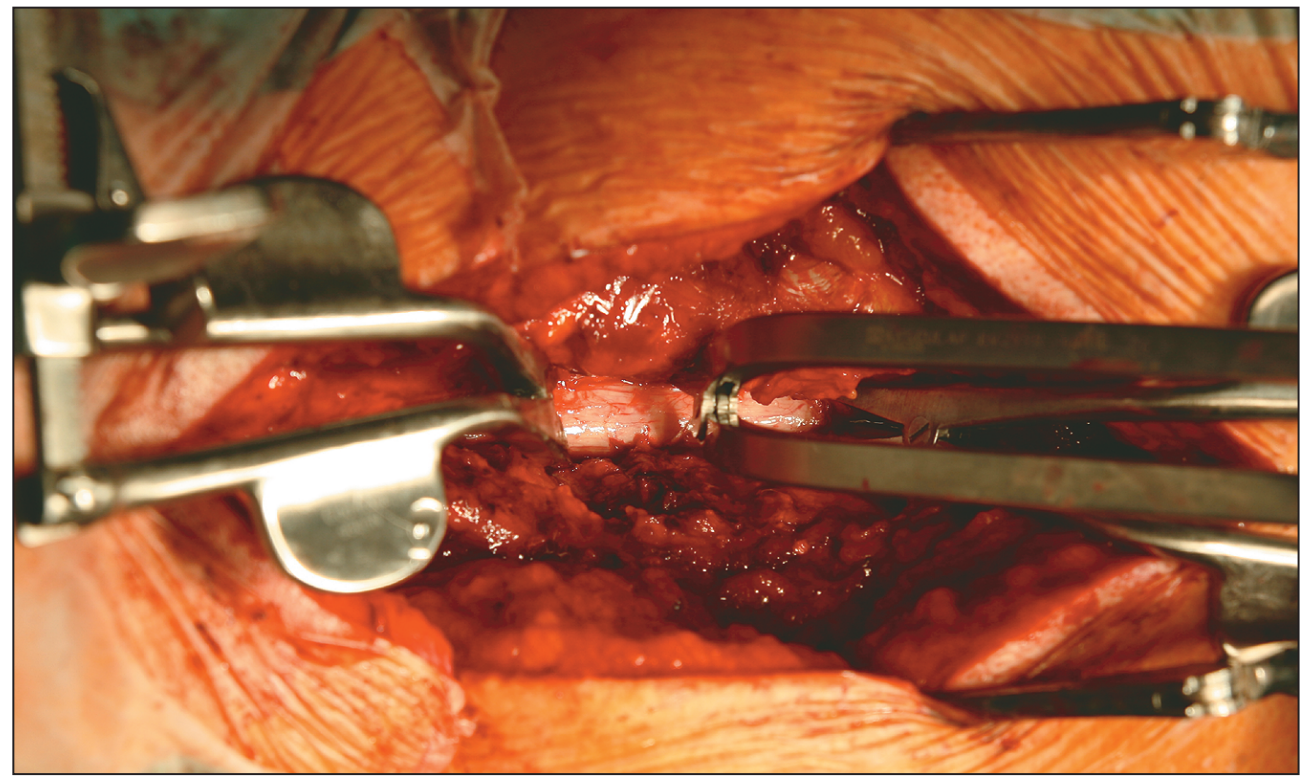

Figure 1. Intraoperative photograph showing the step-by-step distraction of spinous processes and laminas by Cloward-type retractors. The separated and distracted laminae open the operating field and the dura becomes visible 
the end of the retractors to the inner cortex of the vertebral arcus immediately above the dura in the epidural space and to open the retractor with gentle force to prevent the fracture of the spinous process during distraction. Two retractors were applied for each laminae to aid step-by-step distraction of the bone and to prevent its fracture. In the case of intramedullary lesions, the dura was opened and the pathology was removed. If the lesion is visible on the dorsal surface of the cord, a longitudinal posterior midline surgical approach was used with one exception. If the lesion was seen on the posterior surface of the cord, it was approached directly. If the lesion was entirely intraspinal, surgery was performed through a midline exposure. Following removal of space occupying lesions, the dura was partialy closed with or without the use of duraplasty. The narrow operative field and the limited lateral view by the operating microscope make the duraplasty very difficult and time-consuming procedure. In our limited series we left the dural flaps opened in a few cases, and covered the surface with heterologous dural patch and fibrin glue.

In case of the total resection of the intramedullary tumor was not possible due to the lack of recognizable cleavage (diffusely infiltrative tumors) and thus intraspinal space occupation was considered to be solved only temporarily, a bony decompression was indicated to create extra intraspinal space. To avoid the laminae returning to their original position - with the aim of moderate enlargement of the spinal canal - a spacer was placed between the bony parts facing each other (Figure $2 A$ and $B$ ). The space between the distracted laminae was first measured then the appropriate sized tricortical iliac crest bone graft was harvested and inserted between the osteotomized parts of the spinous processes. To avoid the donor site complications we slightly modified the surgical procedure and in 5 cases haemostatic gelatin sponge filled PEEK cages were inserted. We used the

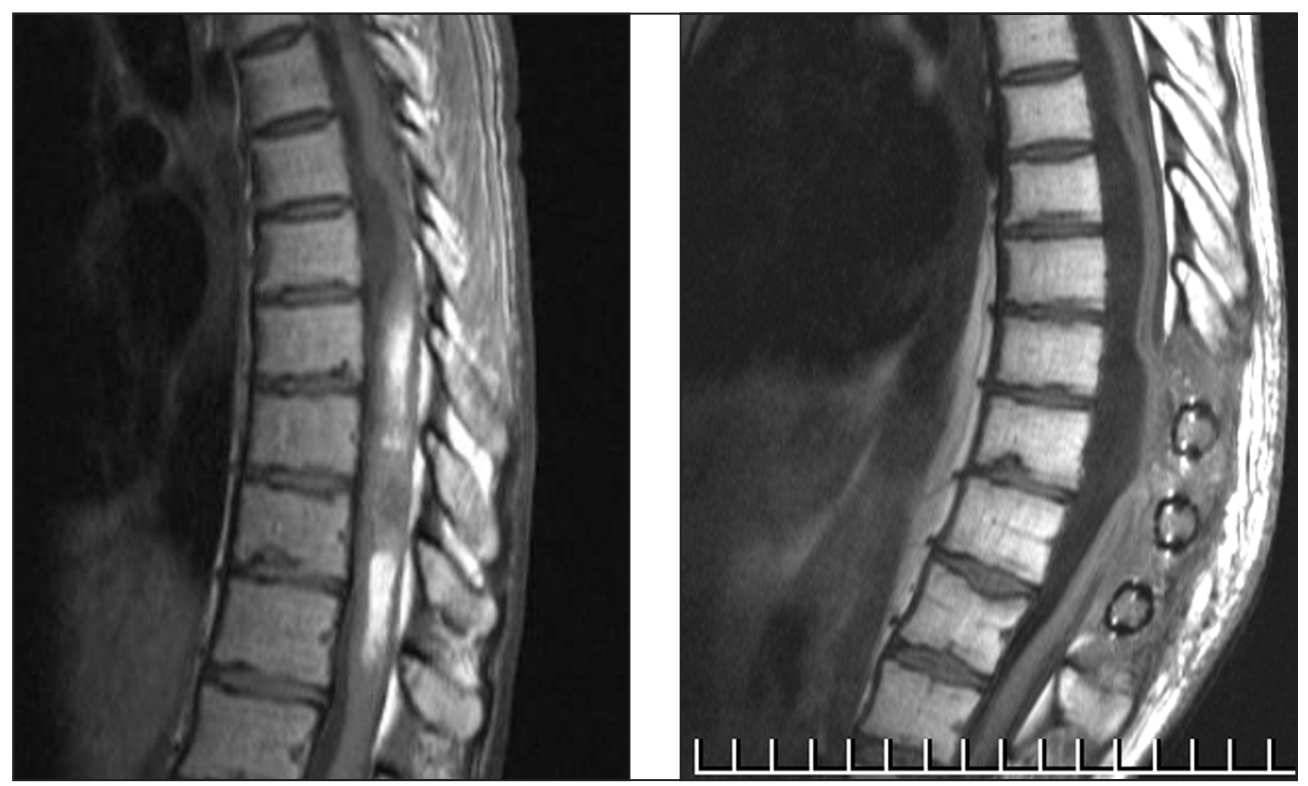

Figure 2. Sagittal T1-weighted magnetic resonance imaging scans showing an intramedullary tumor in the thoracic region before (A) and 12 months after the surgery (B). On the postoperative MRI scan the inserted spacers are also shown 
SOLIS Cervical Cage (Stryker Spine SAS, Z.I Marticot - 33610 Cestas France). This cage has a $\mathrm{D}$ shape design, with $4^{\circ}$ wedge configuration. It has serrations on the top and the bottom face, and incorporates titanium spikes for fixation as well. The cage is available in two footprints, and a variety of heights ranging from $4 \mathrm{~mm}$ to $12 \mathrm{~mm}$. We inserted the cage between the laminae as the plane side of the D shaped cage facing toward the spinal cord, and the convex side of the cage facing outward. The wedge shape of the cage preventing it to sliding out whiles the serrations on the top and bottom side preventing it to sliding into the spinal canal. The strong grasping power of the retracted laminate - returning to their original position - and the two pairs of titanium spikes - located both sides of the cage - fixed it firmly in place. Precise insertion and continuous control of the inner edge of the spacer under the insertion process was necessary to avoid penetration of the graft into the spinal canal, and to avoid compression of the spinal cord.

The bony parts were sutured with Vycril (Ethicon, Inc., Sommerville, NJ) by passing the sutures through the spacer (bone graft or cage) and the holes of the halves created with a small burr. Finally, the fascia and the skin were closed.

\section{Results}

We performed the split laminotomy procedure with or without spacer insertion at various levels of the spine from the level of CIII to the level of LI. The number of split laminae was 3 to 6 . (mean:4) The split spinous processes were closed directly to each other in 24 cases. In 9 cases tricortical bone graft, while in 5 cases PEEK cage insertion was performed.

The average follow-up was 18.7 months, with a range from 7 to 19 months.
Histological results were as follows: 15 intramedullary astrocytomas (Grade I [pilocytic astrocytoma], n:2; Grade II, n:7; Grade III, $\mathrm{n}: 6), 16$ ependymomas, 3 cavernous hemangiomas, 2 dural arteriovenous malformations, one intramedullary arteriovenous malformation, and one hemangioblastoma. The resection of the intramedullary tumor was continued only until that layer where the tumor could clearly be differentiated from the surrounding spinal cord. The completeness of surgical removal depended only on the cleavage plane and not on the approach.

The use of PEEK cages between the osteotomized bony faces requires shorter operative time compared to the classic iliac crest bone grafting method. In our cases the mean duration of the complete surgical procedure was 118 minutes with the range of 91 to 145 minutes. The unnecessary preparation and isolation of the bone graft harvesting area also spare about 12 to 25 minutes.

The mean blood loss was only $110 \mathrm{ml}$ (range, 79-194), as extensive detachment of the muscles, and the second skin incision and iliac bone harvesting was avoided. None of the patients required blood transfusion. No dural tear occurred in our short series. Injury to nervous structures was never observed. No wound infections occurred.

The patients were not braced due to the minimally disturbed anatomy.

The incidence of postoperative local pain was lower, within acceptable limits. (VAS: 2 to 5 ) Furthermore, the patients needed smaller doses of analgesic medications, and early mobilization was allowed. In Group II/B lack of the iliac bone harvesting procedure, no iliac crest pain was detected. The average length of hospital stay was 6 days (ranged 5 to 7 ). 
Results of the preoperative neurological functional assessment in the astrocytoma group were as follow: Grade I, two of all patients, Grade II, twelve of all patients, and Grade III one of the patients. In nine of all patients (60\%) the initial neurological state was unchanged after the surgery, while in six cases (40\%) we detected the worsening of the neurological functions. The ependymoma group patients the initial neurological assessment was: Grade I, fourteen of all patients, Grade II, two of all patients, and Grade III none of the patients. After the surgery we detected improvements of the neurological function in one case (6.25\%). The neurological functions were unchanged in fourteen cases $(87.5 \%)$, and worsening of the symptoms have seen in one patient (6.25\%). The other patients, operated on different lesions had good (Grade I or II) initial neurological functions, and this mostly remained or improved after the surgical procedure (Table 1). The progression of the neurological deficits are due to the malignant (diffusely infiltrative) behavior of the tumor, not the way of the surgical exploration.
To confirm the extension of resection, all patients underwent postoperative MRI evaluations. Neither the inserted homologous bone graft, nor the PEEK cage with the incorporated titanium spikes disturbs the evaluation of the spinal cord on MRI images. Of the 15 astrocytomas, four were removed completely, three were removed subtotally, and eight were partially removed. Of the 16 ependymomas, 13 were removed completely and 3 were partially removed, as confirmed by postoperative MRI scans at 2 months. Cavernous hemangiomas and hemangioblastomas were all completely removed, but the AVM-s were only closed and decompressed. To check bony changes, all patients had postoperative CT imaging. Early postoperative CT scans and $3 \mathrm{D}$ reconstructions showing the split halves of the spinous processes facing each other when no graft was inserted (Group I) (Figure $3 A$ and $B$ ) or the distracted spinous processes and the grafts in-between with the planned slight enlargement of the spinal canal (Group II/A and B) (Figure $4 A$ and $B$ ). Later partial bony healing was seen, mostly in Group I and Group

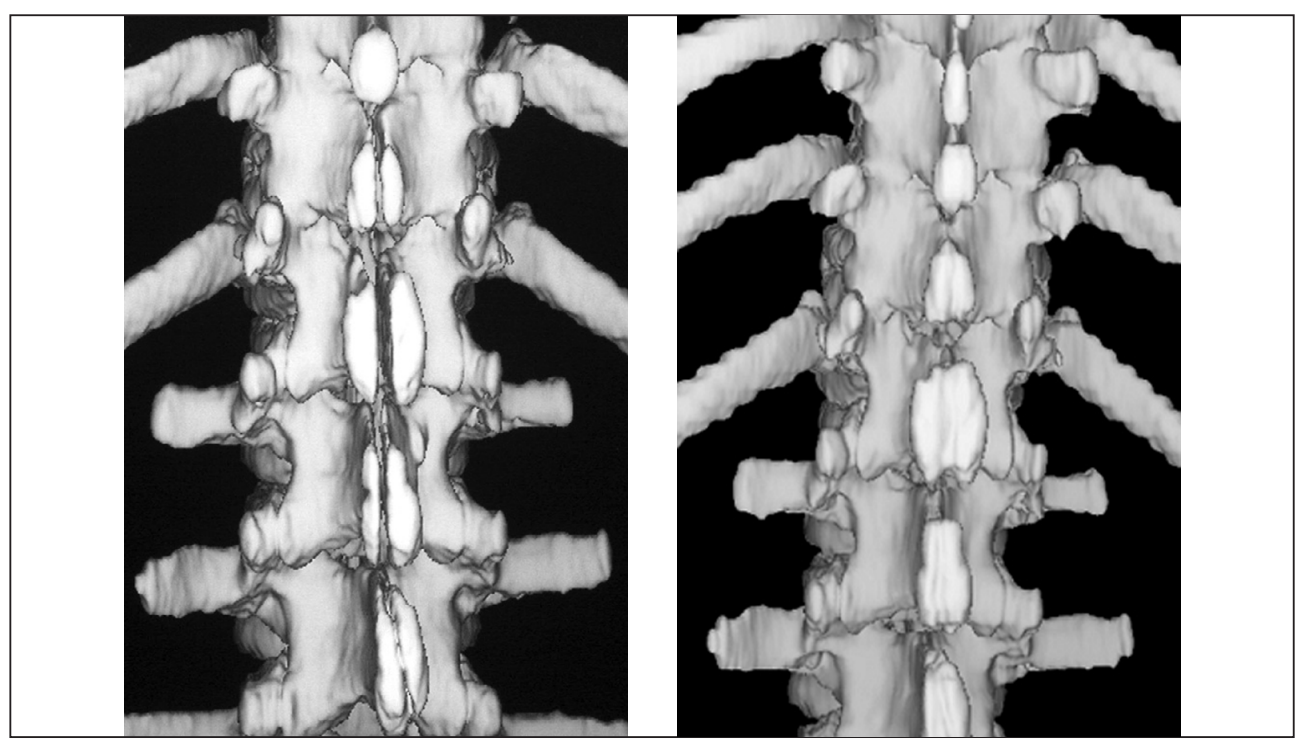

Figure 3. Three-dimensional reconstructed computer tomographic images showing the directly closed spinous processes just after the surgery (A) and bony healing 1 year later (B) 
II/A patients. In Group II (A and B) the permanent distraction of the laminar arches has been followed on CT scans (Figure $5 A$ and $B$ ). Neither graft dislocation nor compression was shown. Some partial fracture of the spinous process was shown on postoperative CT scans in all groups, without clinical significance. Instability was not detected in any of the patients by flexion or extension lateral radiographs.

\section{Discussion}

The surgical approach for treatment of intramedullary tumors has been laminotomy until the last decades. With the aim of preventing the frequently reported postoperative complications various types of surgical techniques have been developed. The main objective of these developments to preserve and reconstruct the posterior spinal structures. The lit-

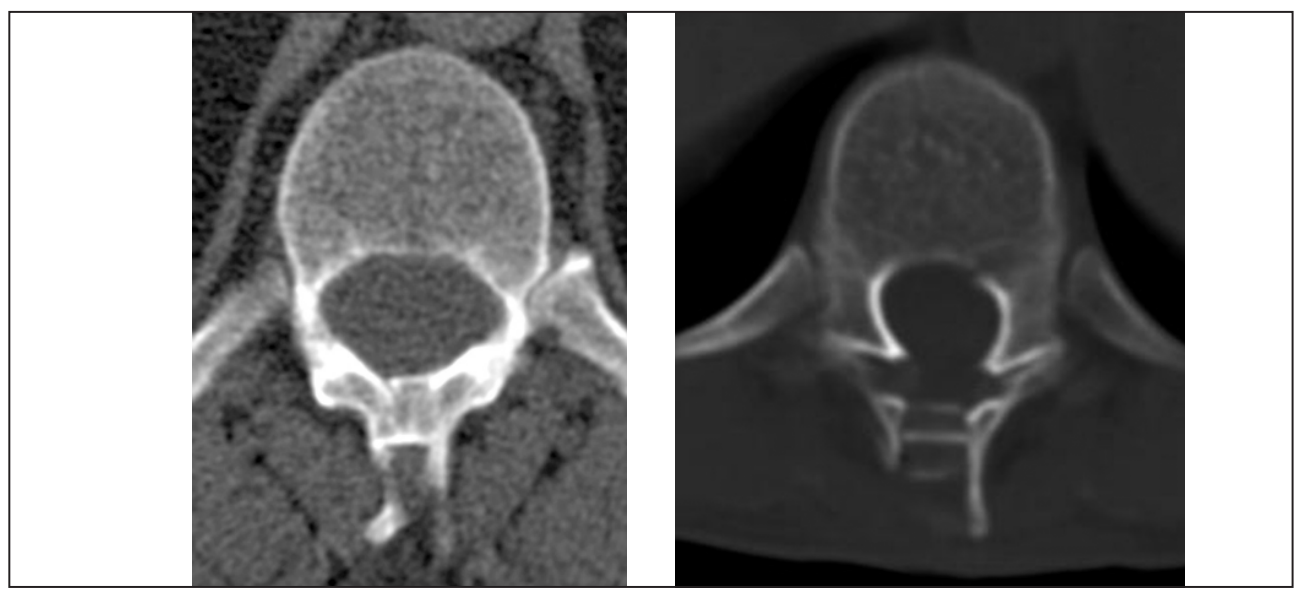

Figure 4. The postoperative axial computed tomographic scans showing the distracted spinal processes, and the position of the bone graft (A), and the PEEK cage (B)
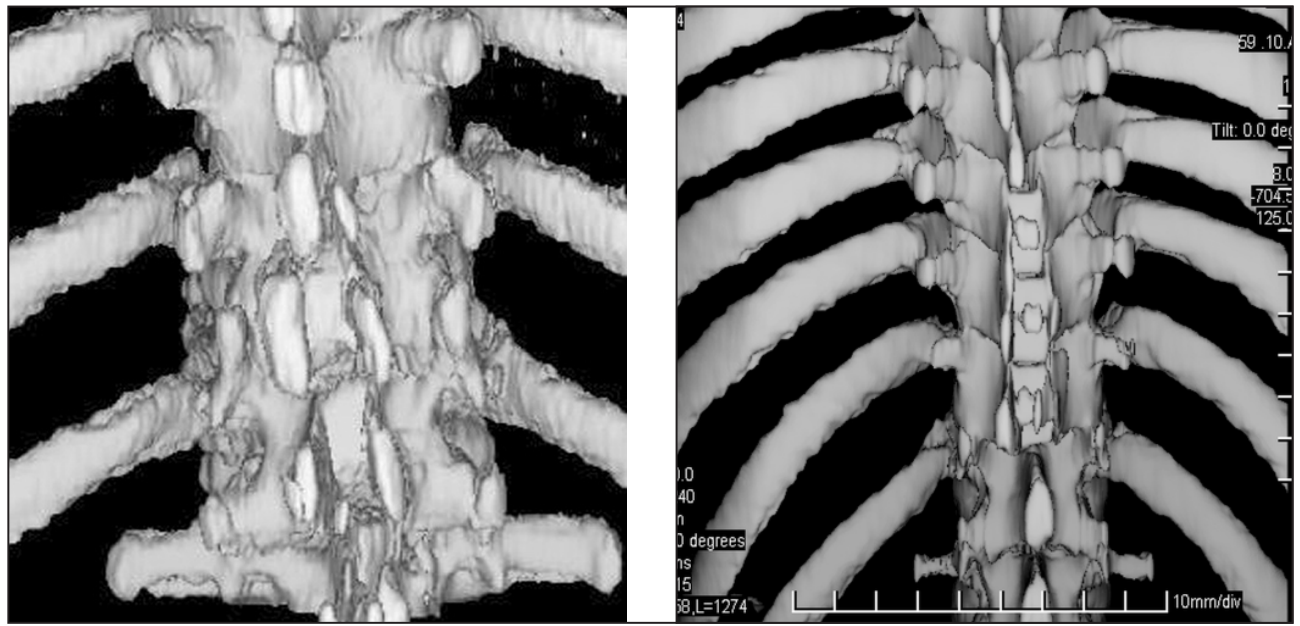

Figure 5. Three-dimensional reconstructed computer tomographic images showing the distracted spinous processes and the positions of tricortical iliac crest bone grafts (A) and PEEK cages (B) after surgery 
erature emphasizes the important role of the deep extensor muscles, the semispinalis, and the multifidus group, especially in the neck. The multifidus and the semispinalis cervicis muscles act as dynamic stabilizers of the cervical spine together with the important static stabilizer structures, including the intervertebral discs, the vertebral arches, ligaments and intervertebral joint capsules. Once these muscles have been detached, it is impossible to reconstruct the complicated anatomy ${ }^{8,10}$. The integrity of the nerves is also important because if they are injured (branches ramified from the dorsal ramus), preservation of the muscles becomes meaningless ${ }^{19}$. The spinous process splitting and distracting laminotomy technique fulfills the requirements of other minimal invasive laminotomy techniques and helps to preserve the attachments of the posterior spinal musculature.

With this method the operative field is restricted compared to laminectomy, but according to the keyhole principle, it is still enough under the operating microscope for the surgery of lesions located within the spinal canal, especially of intramedullary lesions in the midline. Intraoperative identification of the cleavage plane makes removal of intramedullary tumors possible. If there is no recognizable plane in cases of infiltrative or malignant intramedullary tumors (or if intraoperative appearance suggests an infiltrating tumor), tumor removal is not continued at any cost, as this could be dangerous and unnecessary for the patient. If partial tumor removal is performed, or gradually growing residual tumor is expected, bony decompression of the spinal canal is indicated to provide more space. The complementary use of iliac bone graft provide moderate enlargement of the spinal canal at the expense of the postoperative donor site complications. The surgical procedure was modified to achieve the enlargement of the spinal canal by placing heterologous spacer between the fac- ing split bony parts of the spinous process in a way similar to the cervical anterior iliac bone grafting technique. The degree of enlargement of the spinal canal depends on the elasticity of the arches, the force of distraction and the size of the inserted spacer. The press force between the closing laminae and the wedge shape and the serrated face of the cage with the titanium spikes does not allow the spacer to penetrate into the spinal canal or slipping out from the split laminae after its placement. The precise insertion of the bone graft or PEEK cage between the laminae is important, as spacer penetration during the insertion process is a very rare, but possible complication. With this modification of the split laminotomy process, no iliac crest bone graft needed, and all complications of the graft harvesting procedure avoided.

Lack of the most frequent short time postoperative donor site complications, as local pain and hematoma, early recovery and discharge of the patients were possible. The time of the surgical procedure was significantly shorter than the iliac bone grafting procedure. The blood loss during the surgical procedure was also less, compared to the classic iliac bone grafting procedure. Fusion - through the bone grafts or PEEK cages - of the split bony faces of the spinous process was seen in most cases during follow up. There was no patient in whom osseous bridging was missing in all segments.

The bony healing between the osteotomy sites was in agreement with findings of the literature in connection with posterior arch reconstructions of the cervical canal in spondylotic myelopathy cases, and with reconstructions of the laminar roof for a posterior approach. Through our sort follow-up period neither compression, nor displacement of the implanted spacers have been detected. Developing of specially shaped cages for better positioning and distraction of the split laminae need further evaluation. 
It is more difficult to perform a complete distraction of the spinous process and the lamina in adults than in pediatric cases because the spinal arch is less elastic and more fragile. In children, elasticity of the spinal arch is much more pronounced, which allows a wider field for manipulation relative to the diameter of the spinal canal. In the elderly, elasticity is reduced, and fractures are more frequent in patients with osteoporosis. It is easier to distract the relatively thinner and more elastic arches in the cervical region than in the thoracic or lumbar part of the spine. Traumatic bony changes can rarely be observed in the body of the vertebra, mainly in the midline, and fracture of the spinous process can also occur. This had no clinical significance and later showed complete healing. Spinous process distraction may separate the facet joints, but no morphological signs of the destruction or damage of the facet joints or its capsules were observed on CT or MRI scans during the follow-up period, the static stabilizing structures of the spine does not injured. Theoretically, the compliance and elasticity of the spinal arches, facet joints, capsules, and ligaments together allow enough movement under the distraction process to prevent irreversible damage to these structures. In the case of rarely observed overload distraction, these structures moved together, and traumatic bony changes occurred in the midline of the body of the vertebra without clinical significance; they were only seen on CT scan.

The rates of spinal deformities after intraspinal surgery reported in the literature vary considerably. The development of a spinal deformity is a multifactorial process. In our series, no newly developed instability, subluxation, or kyphotic deformity was observed. Open laminectomy produced much greater changes in extension, flexion, and axial rotation than the split laminotomy from the intact. Lateral bending was similarly unaffected for both exposures ${ }^{10}$. Although the clinical and radiological results are very promising, the limited follow-up period precludes conclusions regarding the long-term results of the procedure, especially with respect to kyphotic deformity. The bony protection of the spinal canal and the function of the paraspinal muscles were restored, and we observed better cosmetic results compared with the laminectomy technique. The minimally invasive splitting laminotomy technique allows the incision to be limited to the immediate region of exploration of the spinal canal because, with this method, tissue retraction is minimal and there is excellent access to the affected area. The preservation of the spinous processes and the restoration of the inter- and supraspinosus ligamentous complex maintains the normal posterior median furrow, which is often lost with other, more destructive techniques. Nevertheless, this is a newly developed approach with relatively few cases for each spinal region, and the technique needs further evaluation concerning its limitations. Based on our limited experience, it seems to be safe on all spinal segments (the cervical, thoracic, and lumbar spine) with an acceptable complication rate. Furthermore, it proved to be suitable for removing different, mainly intramedullary spinal pathologies located in the midline. Our novel, modified, minimally invasive technique enables surgeons to obtain a sufficient field for exploring different spinal pathologies that do or do not require spinal canal decompression with preservation of the posterior structures of the spine and the attachments of the muscles.

\section{Conclusions}

The minimally invasive multilevel spinous process splitting and distracting laminotomy approach with or without auto- or allograft (spacer) insertion is a safe and effective surgical management, suitable for removing intra- 
medullary tumors located in the midline of the spinal canal, and ensuring permanent decompression of the spinal cord when necessary. The posterior dynamic and static stabilizing structures of the spine, as the vertebral lami- nae, the joint capsules, ligaments, and the longitudinal musculature are completely prevented. Preservation of these structures helps to avoid the short and long term complications of the widely used laminectomy.

\section{REFERENCES}

1. Banczerowski P, Vajda J, Veres R. Exploration an decompression of the spinal canal using split laminotomy and its modification, the "Archbone" technique. Neurosurgery 2008;62:(5 suppl 2): ONS 432-440.

2. Bartels RH, Donk R, van Azn RD. Height of cervical foramina after anterior discectomy and implantation of a carbon fiber cage. J Neurosurg 2001;95:1 Suppl 40-42.

3. Brooke NS, Rorke AW, King AT, Gullan RW. Preliminary experience of carbon fiber cage prostheses for treatment of cervical spine disorders. Br J Neurosurg 1997;11:221-227.

4. Brotchi J: Intrinsic spinal cord tumor removal. Neurosurg 2002;50:1059-1063.

5. Celik SE, Kara A, Celik S. A comparison of changes over time in cervical foraminal height after tricortical iliac graft or polyetheretherketone cage placement following anterior discectomy. J Neurosurg: Spine 2007;6:10-16.

6. Hosono N, Yonenobu K, Ono K. Neck and shoulder pain after laminoplasty. A noticeable complication. Spine 1996;21:1969-1973.

7. Hukuda S, Ogata M, Mochizuki T, Shichikawa K. Laminectomy versus laminoplasty for cervical myelopathy: brief report. J Bone Joint Surg $(\mathrm{Br})$ 1988;70:325-326.

8. Katsumi Y, Honma T, Nakamura T. Analysis of cervical instability resulting from laminectomies for removal of spinal cord tumour. Spine 1989; 14:1171-1176.
9. Kurokawa T, Tsuyama N, Tanaka H. Enlargement of spinal canal by the sagittal splitting of the spinous process (Jpn). Bessatu Seikeigeka 1982;2:234-240.

\section{Ogdena AT, Bresnahanb L, Smith JS, Natarajand} $R$, Fesslerb RG. Biomechanical comparisons of traditional and minimally invasive intradural tumor exposures using finite element analysis. Clinical Biomechanics 2009;24:143-147

11. Raimondi AJ, Guiterrez FA, Di Rocco C. Laminotomy and total reconstruction of the posterior arch for spinal canal surgery in childhood. J Neurosurg 1976;45:550-560.

12. Rawlinson JN. Morbidity after anterior cervical decompression and fusion. The influence of the donor site on recovery, and the results of a trial of surgibone compared to autologous bone. Acta Neurochir (Wien) 1994;131(1-2):106-118.

13. Ryu SI, Mitchell M, Kim DH. A prospective randomized study comparing a cervical carbon fiber cage to the Smith-Robinson technique with allograft and plating: up to 24 months follow-up. Eur Spine J 2006;15:157-164.

14. Sawin PD, Traynelis VC, Menezes AH. A comparative analysis of fusion rates and donor-site morbidity for autogenic rib and iliac crest bone grafts in posterior cervical fusions. J Neurosurg 1998;88:255-265.

15. Schnee CL, Freese A, Weil RJ, Marcotte PJ. Analysis of harvest morbidity and radiographic outcome using autograft for anterior cervical fusion. Spine 1997;22:2222-2227. 
16. Shad A, Leach JC, Teddy PJ, Cadoux-Hudson TA. Use of the Solis cage and local autologous bone graft for anterior cervical discectomy and fusion: early technical experience. J Neurosurg Spine 2005;2:116-122.

17. Shono Y, McAfee P, Cunningham BW, Brantigan $J W$. A biomechanical analysis of decompression and reconstruction methods in the cervical spine. Emphasis on a carbon-fiber-composite cage. J Bone Joint Surg Am 1993;75:1674-1684.

18. Wilke HJ, Kettler A, Claes L. Primary stabilizing effect of inter-body fusion devices for the cervical spine: an in vitro comparison between three different cage types and bone cement. Eur Spine J 2000;9:410-416.

19. Zhang J, Tsuzuki N, Hirabayashi S, Saiki K. Fujita $K$. Surgical anatomy of the nerves and muscles in the posterior cervical spine: Aguide for avoiding inadvertent nerve injuries during the posterior approach. Spine 2003;28:1379_ 1384.

20. Yasuoka S, Peterson HA, MacCarthy CS. Incidence of spinal column deformity after multilevel laminectomy in children and adults. J Neurosurg 1982;57:441-445.

\section{Zoltán Papp}

National Institute of Neurosurgery H-1145 Budapest, Amerikai út 57.

Tel: (+36) 1 251-2999

Fax: (+36) 1 251-5678 